\title{
Incrementally Discovering Testable Specifications from Program Executions
}

\author{
Neil Walkinshaw and John Derrick \\ Department of Computer Science, The University of Sheffield, Sheffield, UK
}

\begin{abstract}
The ProTest project ${ }^{1}$ is an EU FP7 project to develop techniques that improve the testing and verification of concurrent and distributed software systems. One of the four main work packages is concerned with the automated identification of specifications that could serve as a suitable basis for testing; this is currently a tedious and errorprone manual task that tends to be neglected in practice. This paper describes how this problem has been addressed in the ProTest project. It describes a technique that uses test executions to refine the specification from which they are generated. It shows how the technique has been implemented and applied to real Erlang systems. It also describes in detail the major challenges that remain to be addressed in future work.
\end{abstract}

\section{Introduction}

The ProTest project [1] is motivated by the need to develop improved testing and verification techniques for concurrent and distributed software systems. This paper describes a particular programme of work within the project that aims to reverse-engineer testable models. The work is targeted at systems that are implemented in Erlang [2, and makes use of QuickCheck, the most popular model-based testing framework for Erlang systems.

Erlang is the leading platform for the development of distributed and concurrent software systems. With its Open Telecom Platform (OTP) libraries, Erlang applications can be rapidly developed and deployed across a large variety of hardware platforms. This has caused it to become increasingly popular, not only within large telecoms companies such as Ericsson, but also with a variety of SMEs in different areas. It is increasingly used to develop applications that are business-critical, for example, its use in Ericsson's AXD-301 switch that provides British Telecom's internet backbone.

However, in contrast to the extensive support for rapid program development, the verification and validation of Erlang systems is to-date a largely unsupported. Consequently there is an inherent danger that important functionality remains untested and undocumented. The ProTest project is intended to develop a suite of tools and techniques that help to address this problem.

As a part of this effort, we have developed an automated technique to infer testable specifications from Erlang systems 3 . This does not completely eliminate the need for manual intervention - it has to be inspected and perhaps

\footnotetext{
${ }^{1} \mathrm{http://www} \cdot$ protest-project.eu
} 
slightly amended to represent the correct system - but the required effort is nonetheless substantially reduced. Specifications of the actual system behaviour can be obtained relatively cheaply; they can serve as a useful guide to understanding system behaviour as-is, and can form a suitable basis for regression testing if the current system behaviour is deemed to be correct.

The technique operates by reverse-engineering a specification from traces of program executions. As with most techniques that are based on program traces (referred to as dynamic analysis techniques), the selection of traces is critical [4. One of the key novelties of our technique is that, instead of relying on the developer to record and supply traces, they are obtained automatically by using a model-based test-set generator. The resulting traces are used to refine the model at each iteration, until no further tests that contradict the hypothesised model can be found.

This paper provides details on how the technique works. It shows how the technique has been combined with an Erlang TCP-testing framework to automatically reverse-engineer a model for a Linux TCP implementation. This is followed by a substantial discussion of the main areas that need to be addressed by future work.

\section{Background}

To provide a motivation for our work, we show in section 2.1 how Erlang applications are currently tested with the QuickCheck tool, a commonplace Erlang model-based testing framework. Its weakness is that it currently relies on the developer to supply the models to be tested. An overview of the challenges of reverse-engineering models is given in section 2.2 .

\subsection{Model-Based Testing of Erlang Applications}

QuickCheck [5], an automated model-based testing tool for Erlang. It has become one of the standard testing tools used by Erlang developers. The 'model' is conventionally provided by the developer, as a set of simple properties that must hold for the program to behave correctly, and these have conventionally been expressed as temporal logic properties in Erlang itself. For example, the following property would check that the reverse function for lists behaves as expected:

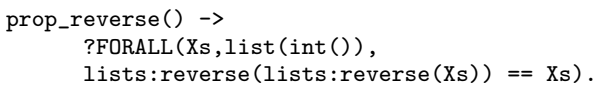

The property prop_reverse generates lots of lists of random length, filled with random integers. For each list (assigned to the variable Xs) it ensures that the list is the same as the reverse of the reverse. Were this not the case, it would have found a bug in the lists:reverse function.

As part of the ProTest project, QuickCheck has been extended to so that it is possible to test an implementation against a finite state machine (rather than 
just a simple property). The use of a finite state machine enables the developer to specify the permitted sequences of program functions, along with their effect on the data-state of the system. QuickCheck tests an implementation by selecting random paths through the state machine, with the aim of verifying their behaviour in the implementation. Given state-machine model, QuickCheck can produce the requisite sequences of inputs (with the necessary data parameters) to automatically test any path in the model against the actual software system (this is important to with respect to our reverse-engineering technique described later).

The key problem with model-based techniques such as QuickCheck is the reliance upon a model that is both accurate and complete. In practice, large and complex systems are often developed under restrictive time-constraints, across multiple sites by different developers, and are constantly evolving. Under such circumstances a developer can at best provide a partial model of dubious accuracy. This is undermined further as the system evolves due to changes in requirements and bug-fixes.

\subsection{Reverse-Engineering State Machines}

Reverse-engineering techniques aim to address this problem. Broadly speaking, these approaches can be separated into two categories: Those based on sourcecode analysis (c.f. [6]), and those based on the analysis of execution traces. Here we focus on the latter (dynamic) approaches. They are based on the analysis of program traces, which are sequences of events (e.g. function calls, messagepassing events etc.), that may optionally be annotated with variable values. The traces can be recorded by instrumenting the source code, or by using tracing tools. For Erlang several such tools are included in the OTP framework.

From a given set of traces, the challenge for reverse-engineering techniques is to produce a candidate state machine that conforms to the provided set of traces. This is not a new problem. Its roots can be traced back to to the 50s, in Moore's work on "gedanken experiments" on state machines [7] and Nerode's work on the synthesis of machines from equivalence relations [8]. However it was Gold's work on Grammar Inference in 1967 that was arguably most influential, establishing the theoretical limits of regular grammar (i.e. deterministic state machine) learnability [9,?]. Most reverse-engineering techniques in the field of software engineering are inspired by techniques that were initially devised as grammar-inference techniques [10[11]12]4].

It is unrealistic to expect an inference technique to be able to infer a machine that is $100 \%$ accurate from any arbitrary set of traces. An inference technique will only produce an accurate result if the provided set of traces is characteristic of the behaviour of the underlying software system [114]. In terms of state machines, this must include enough information about what the program can and cannot do to enable the inference technique to identify every state transition, and to distinguish between every pair of non-equivalent states. Thus the key challenges lie in (a) identifying the relevant subset of executions and (b) collecting them - a potentially expensive and time-consuming process. 
Most current reverse-engineering inference techniques are passive [10[13]14, in that they presume that the necessary traces have already been identified and collected prior to inferring the candidate model. However, given that the initial set of traces is unlikely to contain all of the necessary information, the resulting model is often only poor approximation of the real implementation [4. This problem undermines its practical value; unless the user has an intricate knowledge of the different ways the program can be executed (and has the time to do so), there is no practical way to collect the large volume of traces that are required to produce an accurate model.

\section{An Iterative, Test-Driven Model Inference Approach}

This section describes a specification inference technique that was developed by the authors as part of the ProTest project [3. It circumvents the problems that are intrinsic to conventional passive approaches. It does not rely on a human user to supply it with traces, but instead resorts to a model-based test set generator to identify and collect the traces automatically. The model from which these tests are generated is updated and refined with each iteration, until no more tests can be found that conflict with the model.

The approach is underpinned by the Erlang QuickCheck tool described in section 2.1. The presented technique will infer state machine transition structures, which are represented as Labelled Transition Systems. As usual an LTS ${ }^{2}$ is a quadruple $A=\left(Q, \Sigma, \delta, q_{0}\right)$, where $Q$ is a finite set of states, $\Sigma$ is a finite alphabet, $\delta: Q \times \Sigma \rightarrow Q$ is a partial function and $q_{0} \in Q$.

The rest of this section describes the basic (passive) algorithm that can be used to infer state machines from traces. We use the EDSM Blue-Fringe algorithm [15. This is followed by description of how the EDSM algorithm can be combined with the QuickCheck testing framework, so that traces are automatically extracted from the subject system.

\subsection{Inferring State Machines from Traces}

We begin by describing the underlying algorithm for inferring state machines from traces. State machine inference techniques in the software engineering domain tend to use the $k$-tails algorithm [1016]. This is however prone to several weaknesses (see previous work by the authors [4]). Instead we use the more recent EDSM algorithm that has emerged from the closely related field of regular grammar inference [15].

The EDSM algorithm is presented in algorithm 1 1 . The algorithm works on the basis of two types of trace: a set Pos of traces that represent valid program executions, and a set $\mathrm{Neg}$ of traces that represent either infeasible executions, or executions that terminate with an exception or failur ${ }^{3}$. These traces are first

\footnotetext{
${ }^{2}$ References to "state machines" are henceforth assumed to refer to their LTS.

3 Traditional dynamic analysis approaches presume that all traces represent positive / valid executions. However, in our work, traces that lead to a program failure (i.e.
} 


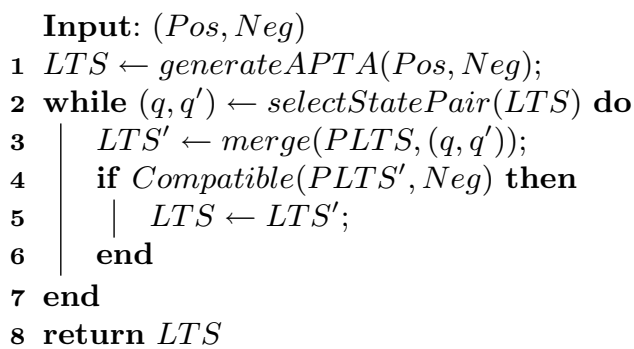

Algorithm 1: EDSM algorithm

arranged into a tree-structured state machine that exactly accepts the given set of traces (this is referred to as an Augmented Prefix Tree Acceptor). In the algorithm this is carried out by the generateAPTA function in line 1 . The process of inferring the state machine subsequently consists of repeating the following process: A pair of states that are deemed to be equivalent is selected, (2) the pair is merged, and (3) the resulting machine is checked against Pos and $N e g$ to ensure that it is still compatible - if not the merge is ignored. The process terminates when no further mergable state-pairs are found.

The key to the effectiveness of the EDSM algorithm lies in the selectStatePair function. It does not simply select the first pair of states that are deemed to be compatible. It computes a "similarity score" for each pair, and then merges pairs in the order of their scores (highest to lowest). The similarity score is computed by counting the extent to which their outgoing paths overlap with each other. The greater the overlap, the greater the score. Thanks to the availability of negative traces, it is also possible to rule-out state merges if they have a conflicting set of outgoing paths (i.e. a sequence is deemed to be possible from one state but not from another). If this is the case, the pair is assigned a score of -1 to prevent a merge from occurring.

The merge function (line 3) takes two states $q$ and $q^{\prime}$, along with the current state machine $A$. In effect, the state $q^{\prime}$ is removed, all of its incoming transitions are routed to $q$ instead and all of its outgoing transitions are routed from $q$. Every time a pair of states is merged, the resulting state machine may be nondeterministic. Nondeterminism is eliminated by recursively merging the targets of non-deterministic transitions.

A comprehensive description of the EDSM algorithm itself is beyond the scope of this paper, and the reader is referred to the original paper by Lang et al. 15] for a more complete description. In practice, our implementation does not compare every pair of states at any given point. It uses a windowing-strategy to compare only those states that are most likely to produce high scores called the "Blue-Fringe" strategy. A detailed description is provided by Lang et al., or in a subsequent survey of grammar inference techniques by Cicchello and Kremer [17.

an exception) are interpreted as "invalid" and so added ot the $N e g$ set in the EDSM algorithm. 


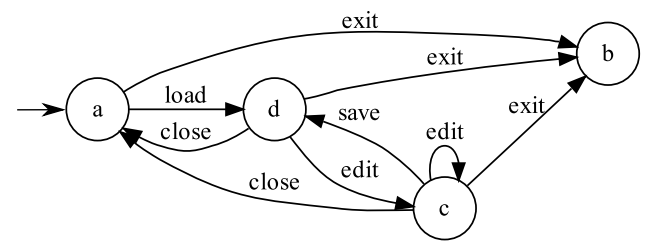

Positive traces:

$<$ load, edit, edit, save, close $>$

$<$ load, edit, save, edit, edit $>$

$<$ load, close, exit $>$

$<$ load, edit, save, edit, save >

$<$ load, edit, save, close, exit >

Negative traces:

$<$ load, close, close >
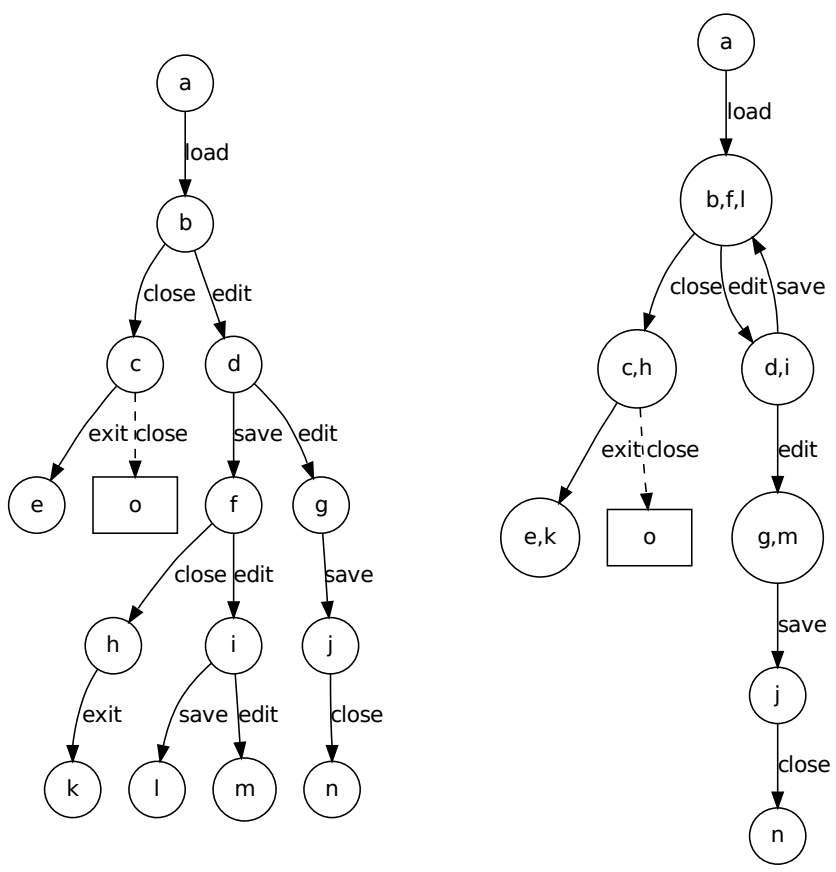

Fig. 1. Prefix Tree Acceptor with added negative trace 
To provide the reader with an intuition of how the algorithm operates, we refer to the example in figure 1. The state machine at the top represents a simple, fictional text editor. The task is to infer it from the set of traces given below, where there are five valid traces, and one trace that has been found to be impossible / invalid. The "prefix acceptor" that is generated from these traces is shown to the right of the traces. The process of selecting state pairs to be merged (as carried out by the selectStatePair function) is illustrated with a selection of examples. Pair $(b, f)$ produce a high score of 5 ; by tracing out the sub-trees from both states there are 5 matching labels in the outgoing sub-trees. On the other hand, pair $(b, c)$ produce a score of -1 , because a "close" is possible from $\mathrm{b}$, but not from c. Any pair that involves node $a$ produces a score of 0 , because a "load" only appears once in the machine and cannot be matched to any other edges in the tree. Thus, we would begin by merging pair $(b, f)$, which would lead to the machine shown to the right of the original prefix tree, and the search begins for the next pair of states to merge.

\subsection{Automating Trace Collection for the EDSM Algorithm with QuickCheck}

When using traces to reverse-engineer state machines (or any other type of model), the key challenge is to supply the technique with a suitable set of traces. The sheer number of traces required, and the prior knowledge and time on the part of the user that is required to execute these traces, often renders the task infeasible in practice [4. The technique that is presented here addresses this problem; it requires no traces from the user, and shows how QuickCheck can be used to automatically gather traces.

Instead of starting with a set of traces, we begin simply with the set of possible inputs or functions that are to label the transitions in the final state machine (i.e. the set $\Sigma$ in the LTS), and also supply the program that is to be tested. Our technique generates the most basic possible state machine from $\Sigma$ - a single state with a loop that is labelled by every element in $\Sigma$. This is translated into a corresponding QuickCheck specification, and QuickCheck attempts to execute a set of random tests that are generated from this general machine. Invariably, tests will disagree with the model; these are recorded and fed into the EDSM algorithm, which produces a more refined model. This is in turn used to generate more tests, which produce a more refined model, and the iterative process continues until QuickCheck can find no further tests that disagree with the model.

The algorithm is displayed in algorithm 2. The notation $L^{L T S}$ refers to the language of the LTS. If a sequence belongs to the language, it represents a sequence of events that should be permitted according to the LTS. The runTest function refers to QuickCheck executing a test, and generateTests refers to the automated random test set generation process implemented by QuickCheck.

A QuickCheck state machine specification consists of more than just an LTS. It corresponds to an extended state machine [18]; for each element in $\Sigma$ it can contain data transformations that manipulate some underlying memory. As it 


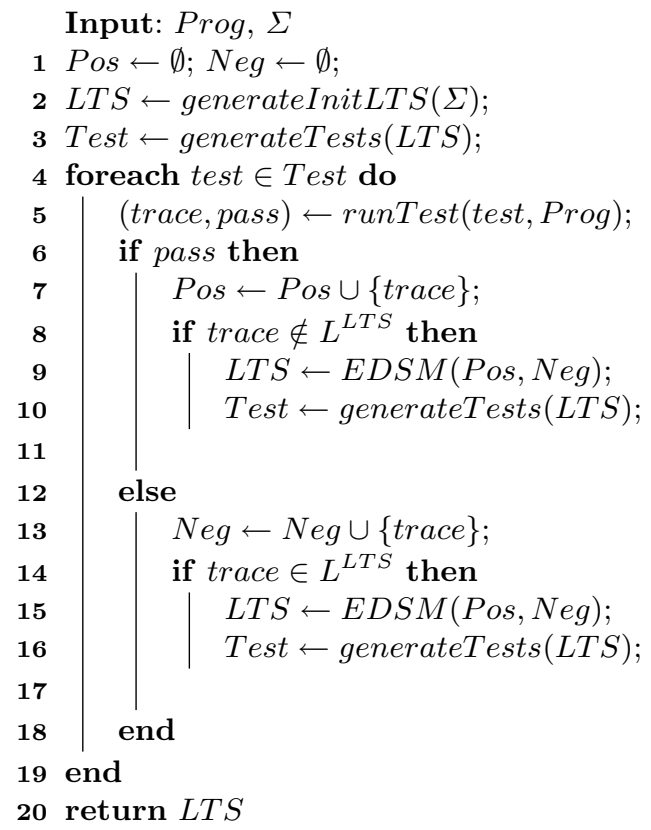

Algorithm 2: InferWithTests

stands, the inference algorithm is restricted to inferring the LTS, and extending it to deal with data-constraints will be discussed in section 5 . In its current form, these elements are supplied by the user in the form of a template. The data-state, along with the way elements in $\Sigma$ change it, is specified by the user and made available as a template file.

The EDSM algorithm has been implemented by the authors in their StateChum tool [12 19]. Traces are recorded by using the comprehensive tracing framework that is built into the Erlang OTP [2. Given the template file generated by the user, a simple Bash script is used to orchestrate the interaction between the QuickCheck testing/tracing process and the StateChum model inference framework.

\section{Case Study - Reverse-Engineering a TCP Stack}

The ProTest project has involved a selection of case studies, to explore the efficacy of the various tools and techniques that are being developed. One of those case studies is a comprehensive QuickCheck testing specification for TCP stacks, which was developed by Paris and Arts [20. To provide a preliminary assessment of our technique, we have applied it to this case-study, with the aim of reverse-engineering an accurate model of a Linux TCP/IP implementation (via a TCP testing extension of the QuickCheck framework). 


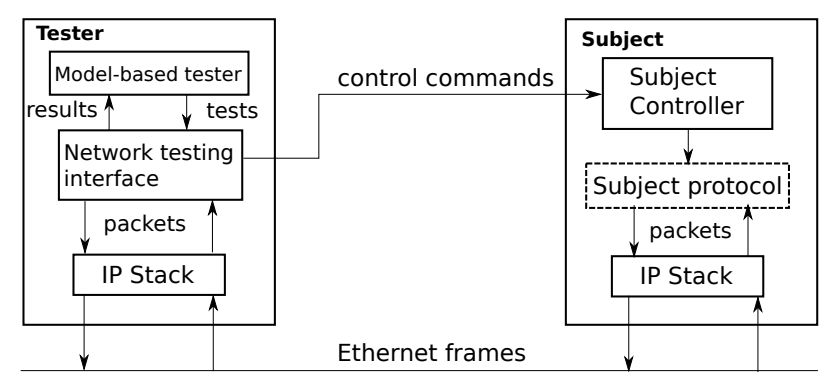

Fig. 2. Communication between tester and subject

Paris and Arts developed a network interface for QuickCheck that enables it to check network protocols on remote machines. The basic process is shown in figure 2. They use two channels to interact with the system under test; one channel is used to induce certain behaviour from the subject (invoking behaviour by invoking functions with Erlang messages), and the other is the network-level channel, which is used to inspect the actual packets that are sent over the network as a result, and to send packets over the network to the subject. The model on the tester machine contains certain constraints that the messages are expected to adhere to, and every time a message is received, it is checked against those constraints. The model then uses the information on these packets to generate valid replies and transition the subject stack through the state machine. Using these two channels enables black box testing of the subject by observing and steering the behaviour using the two interfaces the stack has: to the user through the API (managed through the subject controller), and to the network (using the network channel).

Paris and Arts have used this framework, along with a QuickCheck specification that they have manually generated from the TCP RFC [21]. They have used this as the basis for testing a number of TCP stacks, including the Linux kernel release, as well as a specialised Erlang TCP implementation. As mentioned previously, the state machine is not complete; there are some legal transitions which are not explicitly stated in the graph. There are also many self transitions which are not represented in the graph, but are referred to in the natural language of the RFC. Therein lies the rationale for applying the approach presented here; we want to find the real specification, and not merely an idealized one, so that we have a more authoritative basis for understanding how the system works.

\section{$4.1 \quad$ Results}

Any testing technique for TCP implementations is necessarily limited by the time taken waiting for responses for the server. Some of the operations could take several minutes to execute (mainly due to waiting for time-outs, or packets that may have been lost on the network). Consequently, the process of collecting tests 
was a very time-consuming process. For this reason, the process was limited to 474 iterations (which took 9 hours to collect). In total 1085 tests were executed, 611 of which passed, and 474 of which failed. It is important to bear in mind that this would have been virtually impossible if it was up to a human to collect the traces, and that the diversity of the traces would have been substantially less if the collection process had not been guided by the inference algorithm.

To provide some intuition of the process, the test executions generated for the first 10 test iterations are shown in figure 3 , with the hypothesis model generated for the 10th iteration shown at the bottom and the reference model shown on the right. Failed tests are prefixed with a '-', and passed tests are prefixed with a ' + '. At the beginning, the process starts with the most general LTS, where everything loops to the same state. The first tests are very short, because most of them fail instantly. Nonetheless, these already form a model that has some important commonalities with the reference model; any communication must either start with a listen or an open. An open must be followed by a syn_ack before anything else can happen, and a send_last_ack returns to the initial state.

As more tests are executed, and more possible and impossible sequences are identified, the model is gradually refined. Every test that conflicts with the current model leads to a new, improved model. Depending on the current hypothesis model, QuickCheck will generate tests that exercise scenarios that were not envisaged. In figure 3 the test listen, send_last_ack, listen, passive_close passes (does not cause a program or pre/post-condition failure), even though it should not be possible according to the reference model (also in figure 3). To provide an intuition of the extent of the final set of traces that the technique ended up with, the final APTA is shown in figure 4. Although far too small to read the labels 4 , it does show that the iterative test-generation-and-mode-refinement process was successful at collecting a broad range of program executions.

The final model consists of 38 states, with 277 permitted and 157 forbidden transitions. Structurally, the inferred machine is completely different from the reference model. It is however important to bear in mind that the reference model is only partial - it only describes the small portion of behaviour that is expected, and does not account for what may happen if unexpected sequences of events are produced. For this to be the case it would have to account for every operation at every state. It is this more complete spectrum of behaviour that is represented by the inferred model.

Although more representative of the actual TCP implementation, the inferred model inevitably still contains inaccuracies. The trace-collection process is hampered by the fact that it had to wait for timeouts. More traces would inevitably have led to a more accurate machine. Nonetheless, within the time constraints, the inferred model produced by the EDSM algorithm is the most accurate one possible with current state machine inference techniques.

\footnotetext{
${ }^{4}$ The PDF version of this paper does permit the user to zoom in to read the labels.
} 
1: - send_ack

2: - simultaneous_close

3: - open passive_close

4: - active_close

5: - passive_close

6: - syn_ack

7: - listen active_close

8: - ack_handshake

9: + listen send_last_ack listen passive_close

- listen wait local_close

10: - open simultaneous_close
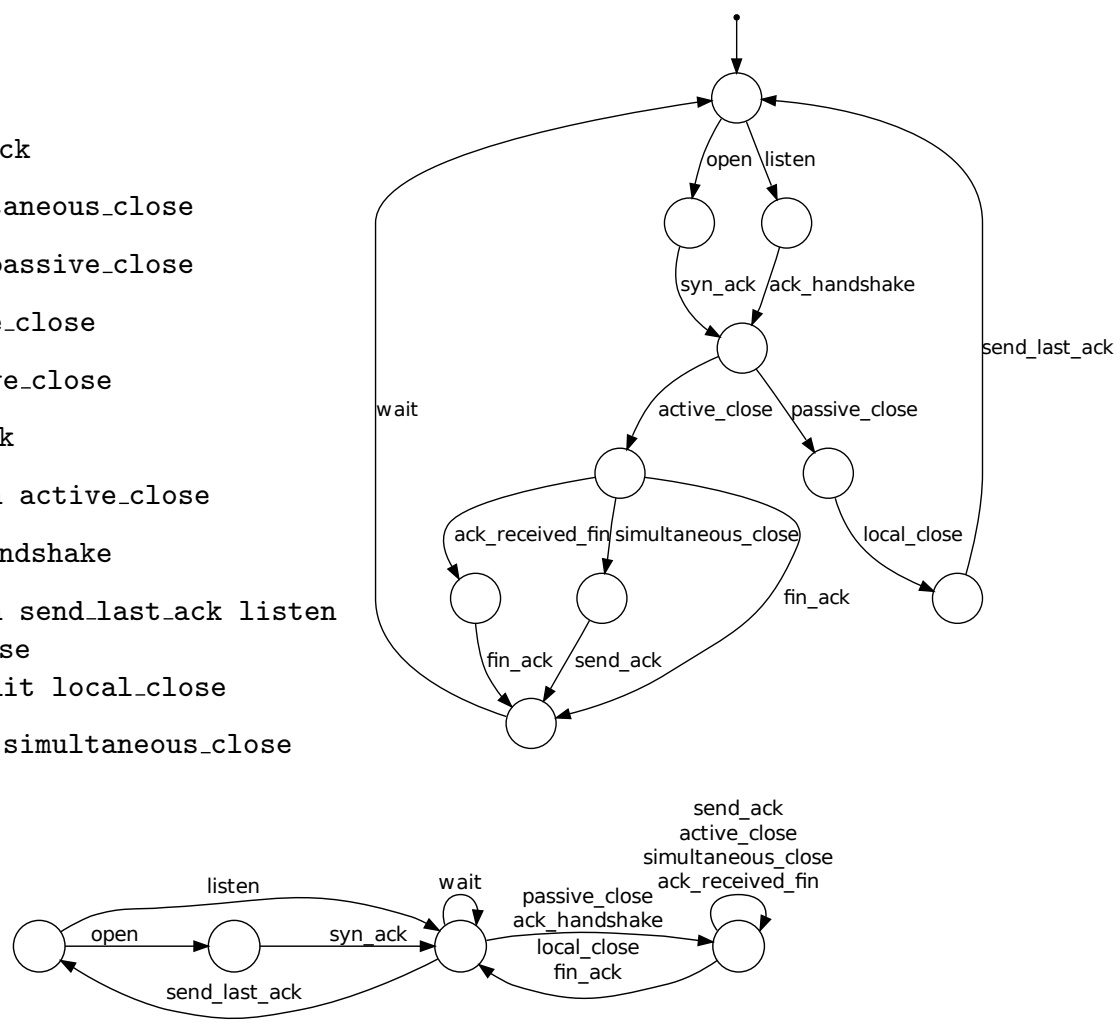

Fig. 3. Test cases for 10 iterations on the left, with the reference TCP model on the right and the hypothesis model after 10th iteration below (Forbidden transitions are omitted for readability)

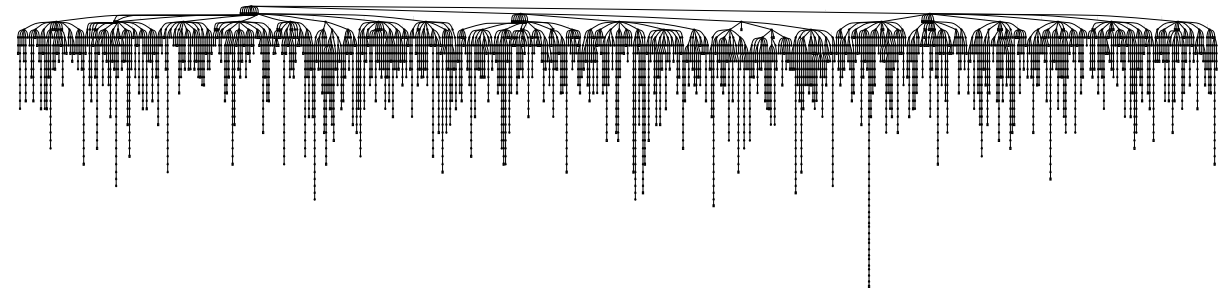

Fig. 4. Final APTA 


\section{Improving the Inference Process}

The presented technique is promising. The combination of QuickCheck with the StateChum model inference framework can seek out the necessary traces, automatically record them, and use them as a basis for inferring a model. All this without the need for interventions from the developer.

There are however still aspects of the technique that do require expertise and effort on the part of the developer. Currently, the QuickCheck template file (minus LTS) has to be generated by hand. This must include any relevant data state information, along with appropriate data transformations. Furthermore, the developer has to manually identify a mapping from the low-level trace events to abstract transition labels in the machine. Both can involve a substantial amount of effort, which we aim to reduce in future work. This section will elaborate on these problems, and will provide some preliminary suggestions for solutions.

\subsection{Inferring Extended State Machines}

The current approach, with the help of the EDSM algorithm, infers labelled transition systems. In practice however, software behaviour cannot be completely described by a simple LTS. In reality, the sequence of events or functions in a program is also dependent upon the memory of the system; a particular function may behave differently (and affect the state of the system in a different way) depending on the state of the system when it is invoked.

To illustrate this problem, we envisage a slightly more sophisticated text editor than the example in figure 1. Imagine that, instead of only being able to open one file at a time, the editor can open and edit multiple files concurrently. This system can no longer be represented in a straightforward LTS with the same labels we use in figure 1. As a simple example, we focus in on the "close" function. As illustrated in figure 5, if multiple files are open, its behaviour ultimately depends on how many files are open; if multiple files are open, it is possible to close one of them and subsequently edit / save / close others. However, if only one file is open, it is subsequently impossible to do any further editing or saving. There are two different ways in which "close" can affect the subsequent behaviour of the software system, and this depends on the underlying state of the system. This more complex, data state-dependent behaviour cannot be represented on a simple deterministic LTS.

There are two approaches that attempt to address this problem. The first approach, which is currently adopted by the authors, is to ensure that functions are chosen in such a way that the different data constraints are encompassed in the labels. The second approach is to adopt a state-labelling procedure that augments states in the PTA or the LTS with data constraints.

5.1.1 Decomposition into atomic functions The decomposition approach works as follows. Instead of using data-sensitive function such as the "close" example above, it can be decomposed into separate sub-functions, based on the 


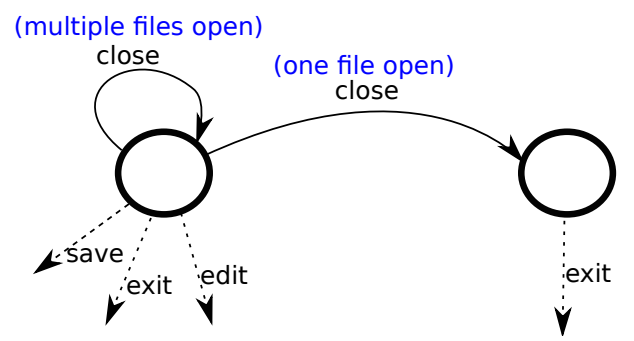

Fig. 5. Behaviour of "close" function in extended text editor

different ways it can affect the system state. The approach is analogous to the Category Partition method used in functional testing [22]. So the "close" function could be divided into "close" and "close_last", making the distinction between the two different types of close behaviour. The data constraints remain implicit, but the data state-based behaviour is represented more accurately by the labels.

This approach places a significant burden on the developer. They have to be aware not only of the individual functions in the system, but also of their potential to change the state of the system. Furthermore, the process of trace collection becomes more intricate. It is no longer sufficient to simply list the functions in the order in which they occur. Every time a data state-dependent function is executed, the data state has to be interpreted, and mapped to the appropriate re-labelled function name that represents the new sub-function. For example, the execution of "close" could no longer be simply listed in the trace as "close", but would have to be mapped to either "close" or "close_last" depending on the current data state.

5.1.2 Labelling states with data constraints Both Dupont et al. 2311] and Lorenzoli et al. 24 describe techniques for integrating data-constraints into the inference process. They deal with different settings; Dupont et al. assume a forward-engineering scenario, where the developer can manually provide the relevant rules for particular sets of states, whereas Lorenzoli et al. describe a reverse-engineering scenario, where they have to mine data constraints from the variable values in a given set of traces.

In the approach proposed by Dupont et al., additional domain knowledge about the system is added in the form of fluents. A fluent is in effect a proposition that is given an initial state, and this state can be altered by a selection of events in $\Sigma$. Given that the relevant fluents are specified for the initial state, the rest of the states in the state machine can be annotated in terms of the fluents by symbolic execution. Consequently, these state annotations can be fed into the inference process, preventing states that correspond to distinct data states from being merged.

Lorenzoli et al. approach the problem from a reverse-engineering angle. In their setting, there is no well-informed developer who can supply suitable fluent- 
like constraints. Instead, they extract data constraints from variable values in execution traces. Data constraints are obtained with the Daikon tool [25], and state comparisons take account of these constraints. Although they describe the approach with respect to the problematic $k$-tails algorithm, there is no reason why it cannot be adapted to other algorithms such as EDSM.

The approach proposed by Lorenzoli et al., of extracting data constraints directly from the traces, is appealing. It provides additional information without increasing the expense in terms of the human effort involved in using the technique. The idea of using data constraint inference to augment the EDSM process forms part of our future work.

Such an approach can however not be applied as-is to large-scale models, derived from realistic traces. Its effectiveness is dependent on the effectiveness of the constraint-inference technique. Daikon (used by Lorenzoli et al.) has some important limitations. It was only designed to identify simple, linear constraints, and any constraint types have to be pre-supplied to the tool.

With respect to our technique, we intend to extend it in the manner of Lorenzoli et al.. To apply to realistic traces, it will necessitate the investigation of more powerful data constraint / function identification techniques - techniques that can identify more relevant complex data transformations, that perhaps incorporate nonlinear variable relationships (c.f. work by Bongard and Lipson on identifying non-linear functions from data [26]).

\subsection{Identifying the Primary Functions in a Trace}

All current reverse-engineering approaches, including those discussed in the previous subsection, make the assumption that the functionas used to label the edges are trivially known (i.e. "edit" and "save"). It is presumed that these functions clearly map to a given trace (i.e. "edit" corresponds to a method in the trace called "edit" etc.). This is fine if the trace contains only a small number of different types of event (such as the traces for the TCP example used above). However, when traces scale up to larger and more complex systems, this becomes impossible. A trace of a trivial Java system for example will often encompass hundreds of thousands calls to thousands of different methods. A simple operation to load a text file might encompass hundreds of different input/output library methods to read the file, and hundreds of font-rendering library methods to render the characters onto the screen for example. Given such a trace, how do we reduce it to a sequence of symbols that can be used to infer a state machine?

To illustrate the problem, we use a simple example of an openly-available Java drawing application called JHotDraw. We may want an abstracted state machine that describes its core functionality. To do so, it is executed and record the trace is recorded: we create three new drawings, and insert five figures into each drawing. The figure in 6 shows the result in JHotdraw.

The problem facing us is this: the ensuing trace contains 161,087 method calls to 1489 different method:5 To reverse-engineer a machine from such a

\footnotetext{
5 The trace was recorded by Cornelissen et al. 27] and can be downloaded from their website.
} 

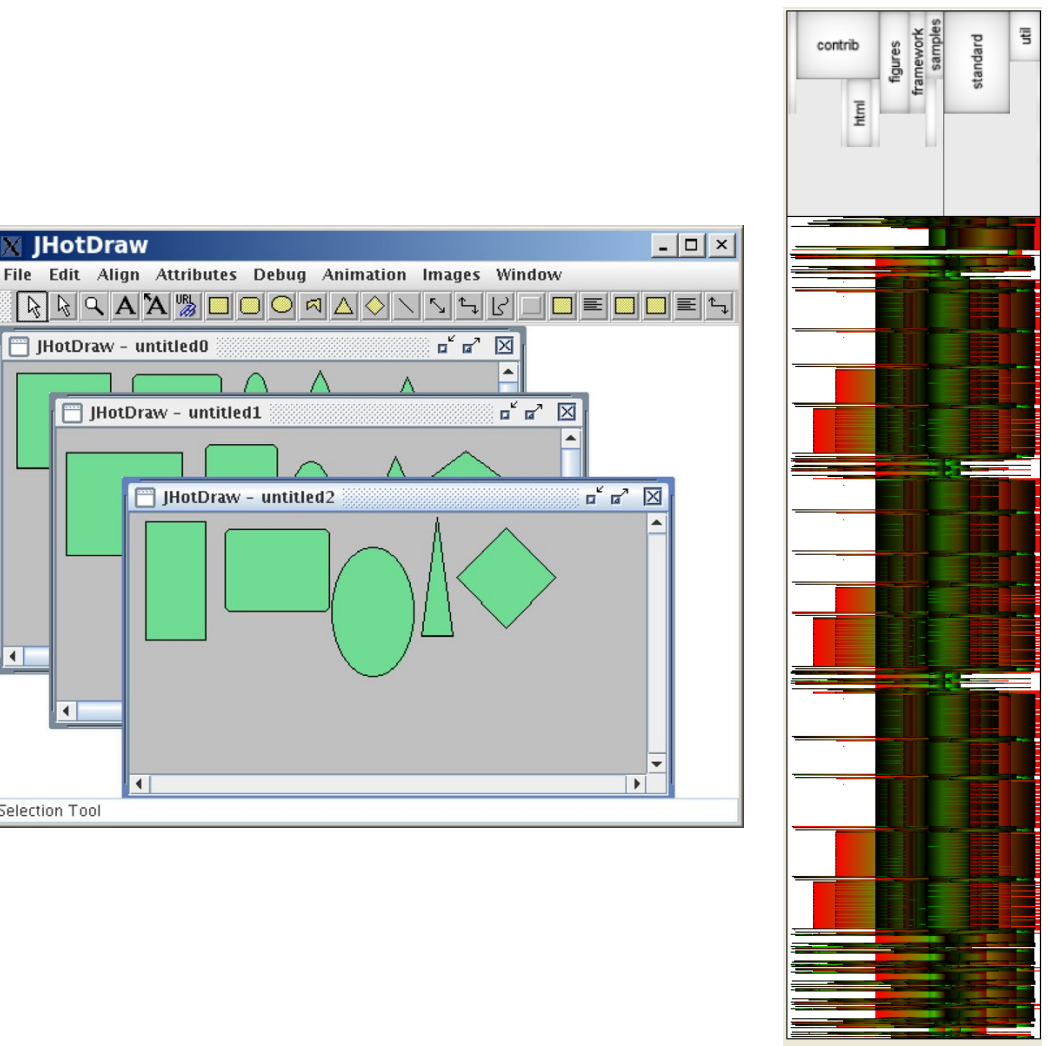

Fig. 6. Screenshot from JHotDraw along with phase-visualisation of the trace [27]

system, we need to map this extremely large trace to a sequence of symbols that will result in a machine that is readable and can be readily understood by the developer. So far, model inference work implies that this is a relatively straightforward process [14; but it can be a very challenging task - especially if the developer is not familiar with the functionality, let alone the architecture of the underlying system.

In practice, a trace is not just an homogeneous series of calls to different methods. It consists of patterns; processing of text file might contain a loop to read in characters that causes the repetition of a few specific methods for a long period of time, followed by a different loop of method calls to display these characters etc. These phases of activity tend to correspond to high-level units of behaviour, and are what we are looking to identify from these traces for our purpose of trace abstraction.

The solution lies in the identification of those 'segments' of the trace that correspond to a particular item of functionality (i.e. rendering a figure). In the 
trace-analysis community this is refered to as phase analysis. Phase analysis is an established problem [27. Current solutions rely on visualisation techniques. The trace is visualised as a vast message sequence chart, which is compressed into a bitmap that fits to the screen. As an example see the trace-visualisation produced by Cornelissen et al. to the right in figure 6. Despite the size of the trace, it is clear that the trace consists of distinct phases of activity. The three repeated actions of drawing figures on the three canvasses is clearly visible in the visualisation.

For the purposes of inferring models however, visualisations are not enough. They only produce bitmaps, as opposed to sequences of symbols that can be used for state machine executions. Visualisations are left to the developer to interpret, and although two phases may look visually identical, they can hide significant differences.

Solving this problem is the focus of ongoing work within the ProTest project. The authors are investigating the application of string compression algorithms such as the LZW algorithm [28 29] and the SEQUITUR algorithm [30. These can automatically identify and label repeated patterns of method invocations, to pull out sequences of high-level functions from a trace, without requiring the expensive and tedious intervention of identifying these functions by hand.

\section{Conclusions}

This paper has presented the challenge of inferring testable models, with a specific focus on testing Erlang systems. The basic reverse-engineering challenge is to find a set of program executions that sufficiently exercise a program to ensure that the reverse-engineered model is accurate. This problem has traditionally been overlooked; developers have supplied sets of traces that are effectively arbitrary; without extensive prior knowledge about the program, it is virtually impossible to collect an adequate set of traces [4.

As part of the ProTest project, the authors have devised a technique [3] that, instead of relying on the manualy supply of traces, automates the trace collection process. It requires a template specification that contains the necessary input values for the functions, but does not need to know the order in which they can be executed. Once this is supplied, it combines a model-based test generation tool [5] with a state machine inference framework [1215] to automatically collect the traces and home-in on a more accurate specification. Its feasibility has been demonstrated with respect to a TCP implementation 20 .

One of the main aims of this paper was to present the main remaining challenges in this area, of which there are several. Current reverse-engineering techniques only have crude approaches to dealing with data-constraints in the model. They also make the big assumption that it is trivial to abstract a program execution trace to a corresponding abstract sequence of symbols. This paper has presented potential avenues of research in these areas, some of which are already being pursued in the context of the ProTest project. 


\section{References}

1. Derrick, J., Walkinshaw, N.: Property-based testing: The protest project. In: FMCO 2009

2. Armstrong, J.: Programming Erlang: Software for a Concurrent World. Pragmatic Bookshelf (July 2007)

3. Walkinshaw, N., Derrick, J., Guo, Q.: Iterative refinement of reverse-engineered models by model-based testing. In: Proceedings of Formal Methods (FM'09). Volume 5850 of LNCS., Springer (2009) 305-320

4. Walkinshaw, N., Bogdanov, K., Holcombe, M., Salahuddin, S.: Improving Dynamic Software Analysis by Applying Grammar Inference Principles. Journal of Software Maintenance and Evolution: Research and Practice (2008)

5. Claessen, K., Hughes, J.: Quickcheck: A Lightweight Tool for Random Testing of Haskell Programs. In: Proceedings of the International Conference on Functional Programming (ICFP). (2000) 268-279

6. Walkinshaw, N., Bogdanov, K., Ali, S., Holcombe, M.: Automated discovery of state transitions and their functions in source code. Software Testing, Verification and Reliability 18(2) (2008)

7. Moore, E.F.: Gedanken-experiments on sequential machines. In Shannon, C.E., McCarthy, J., eds.: Annals of Mathematics Studies (34), Automata Studies. Princeton University Press, Princeton, NJ (1956) 129-153

8. Nerode, A.: Linear automata transformations. Proceedings of the American Mathematical Society 9 (1958) 541-544

9. Gold, E.: Language Identification in the Limit. Information and Control 10 (1967) 447-474

10. Biermann, A., Feldman, J.: On the Synthesis of Finite-State Machines from Samples of their Behavior. IEEE Transactions on Computers 21 (1972) 592-597

11. Dupont, P., Lambeau, B., Damas, C., van Lamsweerde, A.: The QSM Algorithm and its Application to Software Behavior Model Induction. Applied Artificial Intelligence 22 (2008) 77-115

12. Walkinshaw, N., Bogdanov, K., Holcombe, M., Salahuddin, S.: Reverse Engineering State Machines by Interactive Grammar Inference. In: 14th IEEE International Working Conference on Reverse Engineering (WCRE). (2007)

13. Cook, J., Wolf, A.: Discovering Models of Software Processes from Event-Based Data. ACM Transactions on Software Engineering and Methodology 7(3) (1998) $215-249$

14. Ammons, G., Bodík, R., Larus, J.: Mining Specifications. In: 29th SIGPLANSIGACT Symposium on Principles of Programming Languages (POPL), Portland, Oregon (2002) 4-16

15. Lang, K., Pearlmutter, B., Price, R.: Results of the Abbadingo One DFA Learning Competition and a New Evidence-Driven State Merging Algorithm. In: Proceedings of the International Colloquium on Grammar Inference (ICGI). Volume 1433. (1998) 1-12

16. Biermann, A.W., Krishnaswamy, R.: Constructing programs from example computations. IEEE Trans. on Software Engineering SE-2 (1976) 141-153

17. Cicchello, O., Kremer, S.: Inducing grammars from sparse data sets: A survey of algorithms and results. Journal of Machine Learning Research 4 (2003) 603-632

18. Cheng, K., Krishnakumar, A.: Automatic functional test generation using the extended finite state machine model. In: 30th ACM/IEEE Design Automation Conference. (1993) 86-91 
19. Walkinshaw, N., Bogdanov, K.: Inferring Finite-State Models with Temporal Constraints. In: Proceedings of the 23rd International Conference on Automated Software Engineering (ASE). (2008)

20. Paris, J., Arts, T.: Automatic testing of tcp/ip implementations using quickcheck. In: Erlang '09: Proceedings of the 8th ACM SIGPLAN workshop on Erlang, ACM (2009) 83-92

21. Postel, J.: Transmission control protocol. Technical Report 793, DDN Network Information Center, SRI International (September 1981) RFC.

22. Ostrand, T., Balcer, M.: The category-partition method for specifying and generating functional tests. Communications of the ACM 31(6) (1988) 676-686

23. Damas, C., Lambeau, B., Dupont, P., van Lamsweerde, A.: Generating Annotated Behavior Models from End-User Scenarios. IEEE Transactions on Software Engineering 31(12) (2005) 1056-1073

24. Lorenzoli, D., Mariani, L., Pezzè, M.: Automatic generation of software behavioral models. In: ICSE '08: Proceedings of the 30th international conference on Software engineering, New York, NY, USA, ACM (2008) 501-510

25. Ernst, M., Cockrell, J., Griswold, W., Notkin, D.: Dynamically Discovering Likely Program Invariants to Support Program Evolution. Transactions on Software Engineering 27(2) (February 2001) 1-25

26. Bongard, J.C., Lipson, H.: Nonlinear system identification using coevolution of models and tests. IEEE Trans. Evolutionary Computation 9(4) (2005) 361-384

27. Cornelissen, B., Zaidman, A., Holten, D., Moonen, L., van Deursen, A., van Wijk, J.: Execution trace analysis through massive sequence and circular bundle views. Journal of Systems and Software 81(12) (2008) 2252-2268

28. Welch, T.: A technique for high performance data compression. IEEE Computer 17(6) (June 1984) 8-19

29. Afshan, S., McMinn, P., Walkinshaw, N.: Using dictionary compression algorithms to identify phases in program traces. Technical Report CS-10-01, Department of Computer Science, The University of Sheffield (2010)

30. Nevill-Manning, C., Witten, I.: Compression and explanation using hierarchical grammars. Computer Journal 40(2/3) (1997) 103-116 